\title{
Psychological flexibility mediates change in intuitive eating regulation in acceptance and commitment therapy interventions
}

\author{
Essi Sairanen ${ }^{1,2, *}$, Asko Tolvanen ${ }^{1}$, Leila Karhunen ${ }^{3}$, Marjukka Kolehmainen $^{3}$, \\ Elina Järvelä-Reiionen ${ }^{3}$, Sanni Lindroos ${ }^{4}$, Katri Peuhkuri ${ }^{4}$, Riitta Korpela ${ }^{4}$, Miikka Ermes ${ }^{5}$, \\ Elina Mattila ${ }^{4}$ and Raimo Lappalainen ${ }^{1}$ \\ 'Department of Psychology, University of Jyväskylä, Ylistönmäentie 33, PO Box 35, 40014 Jyväskylä, Finland: \\ ${ }^{2}$ Department of Psychology, Karlstad University, Karlstad, Sweden: ${ }^{3}$ Institute of Public Health and Clinical Nutrition, \\ University of Eastern Finland, Kuopio Campus, Finland: ${ }^{4}$ Institute of Biomedicine, University of Helsinki, Helsinki, \\ Finland: ${ }^{5} \mathrm{VTT}$ Technical Research Centre of Finland, Digital Health, Tampere, Finland
}

Submitted 18 August 2016: Final revision received 5 December 2016: Accepted 16 February 2017: First published online 17 April 2017

\begin{abstract}
Objective: Despite the promising results related to intuitive eating, few studies have attempted to explain the processes encouraging this adaptive eating behaviour. The focus of the present study was on exploring mechanisms of change in intuitive eating and weight in acceptance and commitment therapy (ACT) interventions. Mediation provides important information regarding the treatment processes and theoretical models related to specific treatment approaches. The study investigates whether psychological flexibility, mindfulness skills and sense of coherence mediated the interventions' effect on intuitive eating and weight.

Design: Secondary analysis of a randomized control trial. Mediation analysis compared two ACT interventions - face-to-face (in a group) and mobile (individually) - with a control group using a latent difference score model.

Settings: Data were collected in three Finnish towns.

Subjects: The participants were overweight or obese ( $n$ 219), reporting symptoms of perceived stress.

Results: The effect of the interventions on participants' (i) BMI, (ii) intuitive eating and its subscales, (iii) eating for physical rather than emotional reasons and (iv) reliance on internal hunger and satiety cues was mediated by changes in weight-related psychological flexibility in both ACT groups.

Conclusions: These findings suggest that ACT interventions aiming for lifestyle changes mediate the intervention effects through the enhanced ability to continue with valued activities even when confronted with negative emotions and thoughts related to weight.
\end{abstract}

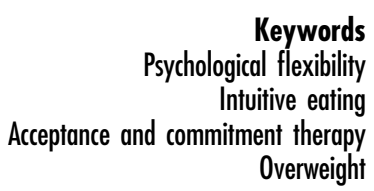

Weight-loss programmes based on restricted eating are becoming more and more popular in social contexts where being slim is perceived as ideal but being overweight has become more common. The long-term benefits of such programmes are questionable, as the majority of individuals eventually regain the weight they lose ${ }^{(1,2)}$. Previous research examining the success of dieting has concluded that diets lead to short-term weight loss, usually $5-10 \%$ of body weight, but this weight loss is not maintained in the long term by the majority of people ${ }^{(1-3)}$. It has been estimated that about $20 \%$ of individuals with overweight are successful at long-term weight loss, when defined as losing at least $10 \%$ of one's initial body weight and maintaining the loss for at least 1 year $^{(4)}$. Most weight-loss diets expect people to constantly control consumption. However, poor long-term results suggest that the majority of persons who have overweight cannot sustain rigid control.

In contrast to controlled eating, intuitive eating is a style of eating that follows the natural contingencies of an individual's perception of physical hunger and satiety cues rather than emotional or environmental cues ${ }^{(5,6)}$. Three central and interrelated components of intuitive eating have been identified: (i) unconditional permission to eat when hungry and what food is desired (i.e. lack of restrictions in eating); (ii) eating for physical rather than emotional reasons; and (iii) reliance on internal hunger and satiety cues to determine when and how much to eat ${ }^{(6)}$. Intuitive eating has found to be related to the absence of eating disorder symptoms ${ }^{(6)}$ and to better 
psychological $^{(6,7)}$ and physical health, such as lower $\mathrm{BMI}^{(6,8,9)}$, cholesterol levels and blood pressure ${ }^{(7)}$. Despite the promising results related to intuitive eating, few studies have attempted to explain the processes supporting intuitive eating regulation. What self-regulation processes are needed in order to resist eating based on emotional or external cues?

Overeating in response to emotional experiences and external cues (e.g. presence of palatable food) can be understood as a failure in self-regulation ${ }^{(10)}$. Studies have indicated that persons with obesity who have difficulty losing or keeping off weight more often use food as a source of comfort and satisfaction ${ }^{(11)}$, eat after difficult interpersonal situations ${ }^{(12)}$ and eat in response to negative emotions $^{(13)}$. Consequently, the function of emotional eating appears to be experiential avoidance that is an attempt to avoid negative private experiences (thoughts, feelings, sensations). Experiential avoidance is a central process in the development of a range of mental health and behavioural health problems ${ }^{(14,15)}$ including eating-related difficulties ${ }^{(16-18)}$. Accordingly, 'third wave' behaviour therapies, such as acceptance and commitment therapy (ACT), focus on avoidance by helping individuals to relate flexibly to interfering private events and regulate their behaviour based on personal values and goals instead of emotional experiences or thoughts ${ }^{(19)}$.

More precisely, ACT is expected to improve individuals' functioning and quality of life by increasing their psychological flexibility including mindfulness skills. Psychological flexibility and mindfulness are adaptive regulation and coping processes that can be seen as opposites to experiential avoidance ${ }^{(20)}$. Psychological flexibility is defined as the ability to act effectively in accordance with one's personal values in the presence of interfering thoughts, emotions and bodily sensations ${ }^{(14)}$. Mindfulness can be described as an adaptive regulation process involving enhanced attention and non-judgemental awareness concerning presentmoment experiences ${ }^{(21,22)}$. The current state of evidence suggests that the concepts specified by the ACT model work very consistently as mediators across the wide range of problems, including eating-related issues ${ }^{(18)}$, targeted by ACT. Successful ACT mediators include general or specific measures of acceptance and psychological flexibility ${ }^{(23-27)}$, defusion $^{(25,28,29)}$, values ${ }^{(25)}$ and mindfulness ${ }^{(16)}$.

Psychological flexibility and mindfulness promote the willingness to approach and experience emotions and are therefore likely to reduce experiential avoidance, such as emotional eating. Mindfulness practice has been suggested to help individuals 'connect' with their inner experiences, such as hunger, thereby attenuating their sensitivity to external or emotional cues to eat ${ }^{(30,31)}$. On a cognitive level, mindfulness has been proposed to reduce identification with thoughts about food, body and shape, thereby interrupting dysfunctional thinking patterns ${ }^{(32)}$ that could predispose someone to emotional or restricted eating. Thus, psychological flexibility and mindfulness can be possible self-regulation processes that promote adaptive eating behaviours, such as intuitive eating, and offer a new approach to effecting weight management.

The current study is based on a recent randomized controlled trial comparing three psychological lifestyle interventions aimed at improving the well-being of adults with overweight experiencing psychological stress: (i) an ACT-based face-to-face group intervention; (ii) an ACT-based mobile intervention; and (iii) a web-based education intervention (for details of the design, see Lappalainen et $a l .{ }^{(33)}$ ). Kolehmainen and colleagues (M Kolehmainen, E Sairanen, E Järvelä-Reijonen et al., unpublished results) found that psychological flexibility related to weight issues improved in ACT intervention groups. The current study sought to further examine the effects of the ACT interventions on psychological processes and adaptive eating behaviour. The aim was to provide a complementary perspective on the role of acceptance and flexibility in the context of intuitive eating by evaluating the mediating effects of mindfulness and psychological flexibility on intuitive eating and weight in terms of improvements. We were interested to see if processes specified by the ACT model worked as mediators in contrast to non-ACT-related processes. Accordingly, the mediating effects of psychological flexibility and mindfulness were investigated in comparison with sense of coherence, a variable less associated with ACT yet commonly used to explain how people manage stressful situations and stay well ${ }^{(34)}$.

The specific aims of the present study were as follows. First, we investigated the effects of the ACT interventions face-to-face (in a group) and mobile (individually) - on intuitive eating, mindfulness and sense of coherence in comparison with each other and a control group. The faceto-face and mobile ACT interventions were expected to have similar effects on all measurements. Intervention effects on weight and psychological flexibility have been reported elsewhere (M Kolehmainen, E Sairanen, E JärveläReijonen et al., unpublished results) and are presented as descriptive statistics herein. Second, we examined whether (i) psychological flexibility (general and weight specific), (ii) mindfulness skills and (iii) sense of coherence mediate the intervention effect on intuitive eating and weight in ACT interventions. The changes in psychological flexibility and mindfulness skills were expected to mediate the intervention effects similarly in both ACT interventions. Psychological flexibility and mindfulness were expected to be more important mediators in the ACT interventions than was sense of coherence.

\section{Methods}

\section{Participants}

The data of the present study stem from a larger lifestyle intervention study ${ }^{(33)}$ that investigated the effects of three low-intensity psychological interventions concerning psychological flexibility, metabolic syndrome risk factors 
and general well-being among individuals with overweight or obesity experiencing stress. The study design and interventions of the larger study are described in detail elsewhere ${ }^{(33)}$ and briefly here. The participants for the study were recruited through advertisements in local newspapers and selected based on specific inclusion criteria: BMI of $27.0-34.9 \mathrm{~kg} / \mathrm{m}^{2}$ (based on self-reported weight and height), age 25-60 years and reporting symptoms of perceived psychological stress (scored at least 3 out of 12 points on the General Health Questionnaire $\left.{ }^{(35)}\right)$. The study took place in Finland.

In the larger study, altogether 298 participants completed the baseline measurements and were randomly divided into an ACT face-to-face intervention group ( $n$ 70), an ACT mobile intervention group ( $n$ 78), a web-based education intervention group $(n$ 79) and a non-treatment control group ( $n$ 71). The participants of both ACT interventions (face-to-face and mobile) and the non-treatment control group comprised the study population of the present study ( $N$ 219; $15.5 \%$ male; $84.5 \%$ female). The web-based education group was excluded from the present study because it could not be compared with the ACT interventions since it had both different content and form of the programme. The mean age of the participants in the present study was 49.5 (SD 7.4) years (range 26.6-60.8 years) and their mean BMI was 31.3 (sD 2.9$) \mathrm{kg} / \mathrm{m}^{2}$ (range $25.7-40.1 \mathrm{~kg} / \mathrm{m}^{2}$ ). About half of the participants had an upper secondary education (51.6\%) and $45.7 \%$ had a university degree. All participants were Caucasian and had Finnish as their native language.

\section{Interventions}

ACT face-to-face group intervention

The ACT face-to-face group intervention consisted of six group sessions during an 8-week period, with each session lasting about $90 \mathrm{~min}$. The intervention programme aimed to support lifestyle changes and to enhance well-being through committed actions based on personally important values. The topics of the six sessions were: (i) my life here and now; (ii) values and mindful living; (iii) value-based actions and barriers; (iv) the observing self and acceptance; (v) mindful eating; and (vi) summary and reflection. Every session included experiential exercises based on the ACT model (such as mindfulness and acceptance exercises and individual activation through value work), pair and group discussions, and homework related to the topic of the session (for a more detailed description of these interventions, see Lappalainen et $\left.a l .{ }^{(33)}\right)$.

\section{ACT mobile intervention}

The participants in the ACT mobile intervention were invited to a group meeting that consisted of a brief overview of ACT principles. In the meeting, the participants were given smartphones that were pre-installed with a stand-alone mental wellness training application ${ }^{(36)}$.
They were instructed to use the application on their own during the 8-week intervention period. The application contained short exercises ( $n$ 41) that teach ACT skills to be applied in daily life. The mobile application delivered an ACT-based intervention programme similar to that of the face-to-face group.

\section{Control group}

The participants randomized into the control group participated in all of the measurements but were not part of any intervention.

\section{Measurements}

Participants completed an Internet-based survey at the pre, post (10weeks after the pre), and follow-up (36weeks after the pre) measurement points of the study. Body weight and height were measured at pre, post and follow-up laboratory visits. The BMI was calculated based on the measured weight and height data.

\section{Intuitive eating}

The Intuitive Eating Scale (IES) ${ }^{(6)}$ is a twenty-one-item instrument containing three subscales that assess the components of intuitive eating: (i) Unconditional Permission to Eat (Permission; nine items, e.g. 'If I am craving a certain food, I allow myself to have it'); (ii) Eating for Physical rather than Emotional Reasons (Reasons; six items, e.g. 'I stop eating when I feel full (not overly stuffed)'); and (iii) Reliance on Internal Hunger and Satiety Cues (Cues; six items, e.g. 'I trust my body to tell me how much to eat'). Participants rated the items on a 5-point Likert-type scale ranging from 1 (strongly disagree) to 5 (strongly agree). The subscale items were averaged, with higher scores indicating higher levels of intuitive eating. The internal consistency of the IES and its subscales was high (Cronbach's $\alpha$ : IES total $=0 \cdot 80$, Permission $=0 \cdot 70$, Reasons $=0.86$, Cues $=0.76$ ).

\section{Mindfulness}

The Five Facet Mindfulness Questionnaire (FFMQ) ${ }^{(37)}$ is a thirty-nine-item measure of being mindful in daily life. It measures the following five elements of mindfulness: (i) Observing (eight items), which includes noticing or attending to internal and external experiences such as sensations, cognitions, emotions, sights, sounds and smells; (ii) Describing (eight items), which involves labelling internal experiences with words; (iii) Acting with Awareness (ActAware; eight items), which represents attending to one's activities of the moment and can be contrasted with behaving mechanically or automatically while attention is focused elsewhere; (iv) Non-judgement of Inner Experiences (NonJudge; eight items), which represents taking a non-evaluative stance towards feelings and thoughts; and (v) Non-reactivity to Inner Experiences (NonReact; seven items), which is the tendency to allow thoughts and feelings to come and go without getting 
carried away by or caught up in them. The items were rated on a 5-point Likert-type scale ranging from 1 (never or very rarely true) to 5 (very often or always true), with higher scores indicating higher levels of mindfulness. The internal consistency of the FFMQ and its subscales was high (Cronbach's $\alpha$ : FFMQ total $=0.91$, Observing $=0.72$, Describing $=0.94, \quad$ ActAware $=0.89, \quad$ NonJudge $=0.90$, NonReact $=0 \cdot 84$ ).

\section{Psychological flexibility}

Psychological flexibility was assessed using the general Acceptance and Action Questionnaire II (AAQ-II) ${ }^{(38)}$ and the Acceptance and Action Questionnaire for Weight $(\mathrm{AAQW})^{(15)}$. The AAQ-II is a seven-item Likert-type questionnaire that assesses the ability to accept aversive internal experiences and to pursue goals in the presence of these experiences. Some items target emotional acceptance or avoidance while others address the tendency to become entangled in thoughts, to take them literally, or, conversely, to see them simply as thoughts; still others ask about the ability to take value-based actions in the presence of difficult thoughts, or about the tendency to become behaviourally inactive or avoidant. The questions of the AAQ-II are based on statements like 'I worry about not being able to control my worries and feelings' and 'My thoughts and feelings do not get in the way of how I want to live my life'. The items were rated on a 7-point Likert-type scale ranging from 1 (never true) to 7 (always true), with higher scores indicating lower levels of psychological flexibility and thus higher levels of experiential avoidance. The internal consistency for the AAQ-II was excellent, with a Cronbach's $\alpha$ of $0 \cdot 91$.

The AAQW ${ }^{(15)}$ is a twenty-two-item Likert-type scale that measures acceptance levels of weight-related thoughts and feelings and the degree to which they interfere with valued actions (e.g. 'I try hard to avoid feeling bad about my weight or how I look'). The items were rated on a 7-point Likert-type scale ranging from 1 (never true/not at all believable) to 7 (always true/completely believable), with higher scores indicating lower levels of psychological flexibility and thus higher levels of experiential avoidance. The questions of the AAQW are based on statements like 'When I evaluate my weight or my appearance negatively, I am able to recognize that this is just a reaction, not an objective fact' and 'I need to feel better about how I look in order to live the life I want'. The internal consistency for the AAQW was excellent, with a Cronbach's $\alpha$ of 0.90 .

\section{Sense of coberence}

Sense of coherence (SOC) was measured with the thirteenitem Orientation to Life Questionnaire (SOC-13) ${ }^{(39,40)}$. The scale consists of three dimensions: Comprehensibility (five items, e.g. 'Do you have very mixed-up feelings and ideas?'), Manageability (four items, e.g. 'How often do you have feelings that you're not sure you can keep under control?') and Meaningfulness (four items, e.g. 'How often do you have the feeling that there's little meaning in the things you do in your daily life?'). The participants were asked to answer the questions on a 7-point semantic differential scale from 1 (never) to 7 (always), with the total sum ranging from 13 (lowest SOC) to 91 (highest SOC). The internal consistency for the SOC-13 was good, with a Cronbach's $\alpha$ of $0 \cdot 87$.

The measures were written in Finnish. The SOC-13 has been indicated to have good validity in Finnish studies ${ }^{(41,42)}$. The IES was translated and back-translated by experts in nutrition and eating behaviour. AAQ-II, AAQW and FFMQ scales were translated by a group of researchers with long experience in acceptance-, mindfulness- and value-based interventions. Previous studies with these measurements have shown comparable results to studies using the original scales ${ }^{(43,44)}$.

\section{Statistical analysis}

The statistical analyses were conducted using the software Mplus version 7 and the statistical software package IBM SPSS Statistics version 20. The parameters were estimated using the full information maximum likelihood method (MLR estimation in Mplus). In the data, missing values (0-12.8\%) were assumed to be missing at random (MAR).

Intervention effects were analysed using the hierarchical linear model (HLM) and effect sizes. Intervention effects, as well as indirect effects, were analysed by first comparing the ACT face-to-face and mobile interventions with each other. If no difference was observed over time between these two ACT intervention groups, then they were combined and together compared with the control group. If the ACT intervention groups showed different effects, then they were separately compared with the control group. The effect sizes were calculated by comparing the mean difference in change $\left(d_{i}-d_{c}\right)$ between the intervention groups (subscript $i$ ) and the control group (subscript $c$ ), using the equation:

$$
\text { Cohen's } d=\frac{d_{i}-d_{c}}{\frac{1}{3} \sum_{j=1}^{3} s d_{j}} ; \quad d_{x}=\left(\bar{y}_{\text {fup }}-\bar{y}_{\text {pre }}\right) ; \quad x=i \text { or } c .
$$

Where:

$$
\begin{aligned}
& \bar{y}_{\text {fup }}=\text { mean of the variable in the follow-up } \\
& \text { measurement; } \\
& \bar{y}_{\text {pre }}=\text { mean of the variable in the pre measurement; } \\
& \frac{1}{3} \sum_{j=1}^{3} s d_{j}=\text { mean of the standard deviation of the pre, } \\
& \text { post and follow-up measurements; and } \\
& s d=\text { standard deviation. }
\end{aligned}
$$

In the first measurement, the difference was divided by the pooled SD. A between-group effect size of 0.2 was considered clinically small, 0.5 medium and 0.8 large ${ }^{(45)}$.

The mediation analyses were conducted using the latent difference score (LDS) mediation model $^{(46)}$. Figure 1 shows the LDS model, where the effects of the ACT interventions on intuitive eating (change from pre to 


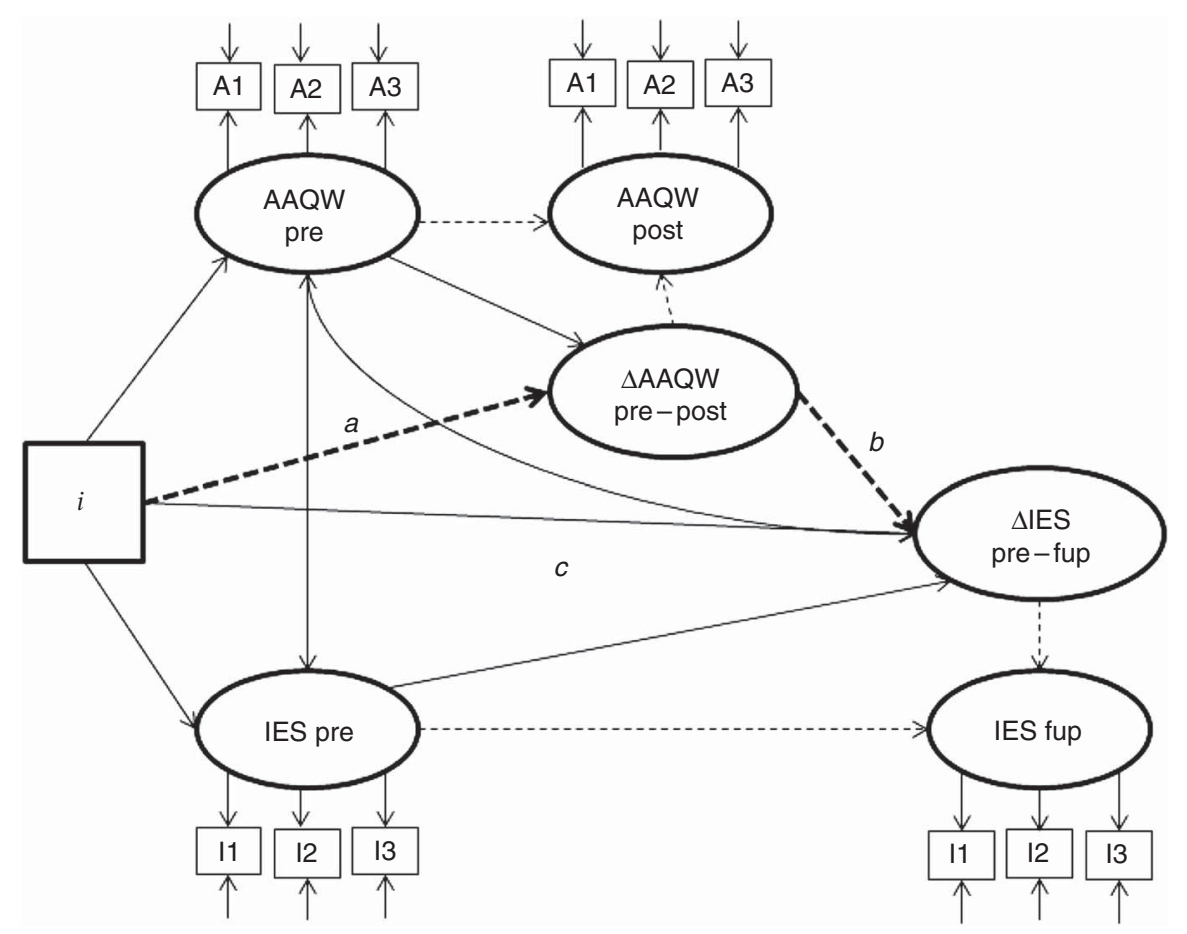

Fig. 1 The latent difference score mediation model, where the intervention effect on the IES (change from pre to fup) is mediated by the AAQW (change from pre to post). Note: $i=0$ for control group; $i=1-2$ for intervention groups (IES, Intuitive Eating Scale; AAQW, Acceptance and Action Questionnaire for Weight; pre, pre-intervention; post, post-intervention; fup, follow-up; regression coefficients marked as - . - - > in the model are fixed to 1)

follow-up, $\Delta$ IES) are mediated by the change in psychological flexibility during the interventions as assessed with the AAQW ( $\triangle \mathrm{AAQW})$. The product of the $a$ and $b$ coefficients in the LDS model comprises the mediation effect. In the measurement model, three parcels (e.g. A1-A3) were used to estimate the latent factors representing the true score without measurement error. By constructing three measured indicators (parcels) for each latent variable (i.e. psychological flexibility and intuitive eating), we followed the recommendation of Russell et $a l .{ }^{(47)}$.

The LDS model was chosen because the focus was on variance in within-individual changes in true scores and on mean intervention effects. The LDS approach made it possible to focus on change (e.g. $\triangle \mathrm{AAQW}$ ) in each construct rather than only on levels (e.g. AAQW). Thus, we are suggesting that changes in behavioural processes (psychological flexibility, mindfulness skills and sense of coherence) are more important than, for example, psychological flexibility per se.

We followed the recommendation of Zhao et al. ${ }^{(48)}$ in the analysis of mediation. They have presented that the only requirement to demonstrate mediation is a significant indirect effect $(a \times b$, see Fig. 1). It may be possible to establish an indirect effect despite no total direct effect ${ }^{(48)}$. The product of $a$ and $b$ may be significant even if the coefficients on their own are not ${ }^{(49)}$. Mplus allows users to define any function of parameters $(a \times b)$ as a model parameter and in addition provides bias-corrected bootstrap CI for such parameters. CI are based on 1000 bootstrap resamples. Indirect effects are deemed statistically significant at the 0.05 level if the $95 \% \mathrm{CI}$ for the estimate of indirect effects does not include zero.

The fit of the models was evaluated using the following goodness-of-fit measures provided by the Mplus program $^{(50)}$ : RMSEA (root-mean-square error of approximation, with values of 0.06 or less indicating a good fit); SRMR (standardized root-mean-square residual, with values less than 0.08 indicating a good fit); CFI (Bentler's comparative fit index, with values of 0.95 or higher indicating a good fit); and TLI (Tucker-Lewis index, with values higher than 0.95 indicating a good fit) ${ }^{(51)}$.

\section{Results}

\section{Intervention effects}

Changes in intuitive eating and weight during the interventions and at the 6-month follow-up measurement point are described in Table 1. Eating for Physical rather than Emotional Reasons, a component of intuitive eating, increased significantly in the ACT face-to-face and mobile groups compared with the control group $(P=0.003)$. The increase was significant from the pre- to post-intervention measurement $(P=0.001)$, but not from the postintervention to follow-up measurement $(P=0.718)$. The interventions did not have significant effects on the other two subscales of intuitive eating (Unconditional Permission to Eat and Reliance on Internal Hunger and Satiety 
Table 1 Changes in outcome measurements for the acceptance and commitment therapy (ACT) intervention (face-toface and mobile) groups and control group. Between-group effect sizes and between-group analyses compare ACT interventions with the control group

\begin{tabular}{|c|c|c|c|c|c|c|c|c|}
\hline \multirow[b]{2}{*}{ Variable } & \multicolumn{2}{|c|}{ Pre } & \multicolumn{2}{|c|}{ Post } & \multicolumn{2}{|c|}{ Fup } & \multicolumn{2}{|c|}{ Change pre-fup } \\
\hline & Mean & SD & Mean & SD & Mean & SD & $\begin{array}{l}\text { Between-group } \\
\text { effect size, } \\
\text { Cohen's } d\end{array}$ & $\begin{array}{l}\text { Between-group analysis: } \\
\text { ACT (face/mobile) } \\
\text { v. control, } P \text { value }\end{array}$ \\
\hline \multicolumn{9}{|l|}{ IES total } \\
\hline Face & 2.9 & 0.4 & 3.0 & 0.5 & $3 \cdot 1$ & 0.5 & 0.43 & 0.109 \\
\hline Mobile & 2.9 & 0.5 & $3 \cdot 1$ & 0.4 & $3 \cdot 1$ & 0.5 & 0.43 & \\
\hline Control & 3.0 & 0.5 & 3.0 & 0.5 & 3.0 & 0.5 & & \\
\hline \multicolumn{9}{|c|}{ IES: Permission } \\
\hline Face & 3.0 & 0.5 & 3.0 & 0.5 & $3 \cdot 1$ & 0.5 & 0.18 & 0.820 \\
\hline Mobile & $3 \cdot 1$ & 0.6 & $3 \cdot 2$ & 0.6 & $3 \cdot 1$ & 0.7 & 0.00 & \\
\hline \multirow{2}{*}{\multicolumn{9}{|c|}{ IES: Reasons }} \\
\hline & & & & & & & & \\
\hline Face & $2 \cdot 4$ & 0.8 & $2 \cdot 6$ & 0.7 & $2 \cdot 8$ & 0.9 & 0.36 & $0.003^{\star \star}, \dagger$ \\
\hline Mobile & $2 \cdot 4$ & 0.8 & 2.6 & 0.8 & $2 \cdot 7$ & 0.8 & 0.24 & \\
\hline Control & $2 \cdot 6$ & 0.9 & $2 \cdot 6$ & 0.8 & $2 \cdot 7$ & 0.8 & & \\
\hline \multicolumn{9}{|l|}{ IES: Cues } \\
\hline Face & $3 \cdot 2$ & 0.6 & 3.3 & 0.7 & 3.4 & 0.6 & 0.15 & 0.970 \\
\hline Mobile & $3 \cdot 2$ & 0.7 & $3 \cdot 3$ & 0.6 & 3.4 & 0.6 & 0.15 & \\
\hline Control & $3 \cdot 2$ & 0.7 & $3 \cdot 3$ & 0.6 & $3 \cdot 3$ & 0.7 & & \\
\hline \multicolumn{9}{|c|}{ Weight (kg) } \\
\hline Face & $86 \cdot 1$ & $10 \cdot 2$ & $85 \cdot 3$ & $10 \cdot 1$ & 84.4 & $11 \cdot 0$ & $-0 \cdot 10$ & $0 \cdot 177$ \\
\hline Mobile & 88.4 & $10 \cdot 3$ & 88.0 & $10 \cdot 4$ & $87 \cdot 2$ & 11.0 & -0.06 & \\
\hline Control & $88 \cdot 3$ & 11.4 & $88 \cdot 2$ & $11 \cdot 6$ & $87 \cdot 7$ & $11 \cdot 8$ & & \\
\hline \multicolumn{9}{|c|}{ BMI $\left(\mathrm{kg} / \mathrm{m}^{2}\right)$} \\
\hline Face & 31.0 & $3 \cdot 1$ & $30 \cdot 7$ & 3.2 & $30 \cdot 3$ & 3.4 & -0.17 & 0.145 \\
\hline Mobile & $31 \cdot 6$ & $2 \cdot 7$ & 31.5 & $2 \cdot 8$ & $31 \cdot 1$ & 2.9 & $-0 \cdot 10$ & \\
\hline Control & $31 \cdot 2$ & $2 \cdot 8$ & $31 \cdot 1$ & $2 \cdot 8$ & $31 \cdot 0$ & 3.0 & & \\
\hline
\end{tabular}

Pre, pre-intervention; post, post-intervention; fup, follow-up; IES, Intuitive Eating Scale; Permission, Unconditional Permission to Eat subscale; Reasons, Eating for Physical rather than Emotional Reasons subscale; Cues, Reliance on Internal Hunger and Satiety Cues subscale.

Means and SD were calculated using Mplus software version 7 with full information maximum likelihood estimates for the parameters. A between-group effect size of 0.2 was considered clinically small, 0.5 medium and 0.8 large $^{(45)}$.

${ }^{\star *} P<0.01$.

†Difference in change scores from pre to post was significant at the $P<0.05$ level. Difference in change scores from post to fup was not significant at the $P<0.05$ level.

Cues), nor on the total score of the IES or on weight in comparison with the control group.

Changes in process measurements during the interventions and their follow-up are described in Table 2. Psychological flexibility for weight (AAQW) improved significantly in the ACT face-to-face and mobile groups during the respective interventions compared with the control group $(P<0.001)$. In addition, participants' tendency for Observing (subscale of FFMQ) increased significantly in both ACT groups in comparison to the control group from the pre- to the post-intervention measurement $(P=0 \cdot 001)$.

Mindfulness skills (FFMQ total) and its subscales Acting with Awareness and Non-reactivity to Inner Experiences improved more in the ACT mobile group than in the ACT face-to-face group $(P=0.018,0.021$ and 0.043 , respectively). Accordingly, they were separately compared with the control group. In the ACT mobile group, the FFMQ total increased significantly more compared with control from the pre- to the post-intervention measurement $(P=0.002)$. Changes in Acting with Awareness and Non-reactivity to Inner Experiences were not significant in either of the ACT groups compared with the control group.

\section{Mediation analysis}

We were interested in whether changes in process variables (AAQ-II, AAQW, SOC-13, FFMQ and its subscales) during the active intervention period (from pre to post) mediated the long-term changes (from pre to follow-up) in intuitive eating and weight. All LDS mediation models showed an excellent fit to the data (RMSEA $=0 \cdot 000-0 \cdot 072, \quad$ SRMR $=$ $0 \cdot 030-0 \cdot 072$, CFI $=0.974-1.000$ and TLI $=0.959-1 \cdot 014)$.

The estimates and $95 \%$ CI of standardized indirect effects $(a \times b)$ in the LDS models are described in Table 3 (see Fig. 1). There were no significant differences in indirect effects between the ACT face-to-face group and the ACT mobile group; subsequently, the ACT groups were compared jointly with the control group. The $95 \% \mathrm{CI}$ for indirect effects - via the AAQW - on (i) weight (BMI), (ii) intuitive eating total and its subscales, (iii) Eating for Physical rather than Emotional Reasons and (iv) Reliance on Internal Hunger and Satiety Cues did not include zero. Thus, the LDS models suggested that the effect of the ACT interventions on those variables was mediated by the change in weight-related psychological flexibility (AAQW). The unstandardized regression coefficients $(a, b$ and $c$ ) of the significant mediation models are 
Table 2 Changes in process measurements for the acceptance and commitment therapy (ACT) intervention (face-toface and mobile) groups and control groups. Between-group effect sizes and between-group analyses compare ACT Interventions with the control group

\begin{tabular}{|c|c|c|c|c|c|c|c|c|}
\hline \multirow[b]{2}{*}{ Variable } & \multicolumn{2}{|c|}{ Pre } & \multicolumn{2}{|c|}{ Post } & \multicolumn{2}{|c|}{ Fup } & \multicolumn{2}{|c|}{ Change pre-fup } \\
\hline & Mean & SD & Mean & SD & Mean & SD & $\begin{array}{l}\text { Between-group } \\
\text { effect size, } \\
\text { Cohen's } d\end{array}$ & $\begin{array}{l}\text { Between-group analysis: } \\
\text { ACT (face/mobile) } \\
\text { v. control, } P \text { value }\end{array}$ \\
\hline \multicolumn{9}{|l|}{ AAQ-II } \\
\hline Face & $20 \cdot 2$ & 8.5 & $18 \cdot 1$ & $8 \cdot 4$ & $17 \cdot 0$ & $7 \cdot 6$ & -0.12 & 0.233 \\
\hline Mobile & $20 \cdot 3$ & $9 \cdot 1$ & $18 \cdot 6$ & $9 \cdot 0$ & $16 \cdot 0$ & 8.4 & -0.25 & \\
\hline \multirow{2}{*}{\multicolumn{9}{|c|}{ AAQW }} \\
\hline & & & & & & & & \\
\hline Face & 85.4 & $19 \cdot 2$ & 78.0 & $19 \cdot 0$ & 73.0 & $21 \cdot 1$ & -0.44 & $0.000^{\star *}, \dagger$ \\
\hline Mobile & $88 \cdot 2$ & $21 \cdot 1$ & $80 \cdot 9$ & $19 \cdot 9$ & 80.5 & $22 \cdot 7$ & -0.20 & \\
\hline Control & $87 \cdot 3$ & $20 \cdot 1$ & 86.0 & 22.4 & 83.7 & 23.2 & & \\
\hline \multicolumn{9}{|c|}{ FFMQ total } \\
\hline Face & $134 \cdot 7$ & $17 \cdot 0$ & $134 \cdot 0$ & $19 \cdot 5$ & $139 \cdot 0$ & $18 \cdot 8$ & 0.06 & $0.967 \ddagger / 0.009^{\star \star}, \dagger, \S$ \\
\hline Mobile & 131.7 & $19 \cdot 1$ & 137.3 & $19 \cdot 3$ & 139.7 & 21.5 & 0.13 & \\
\hline Control & $131 \cdot 1$ & $20 \cdot 4$ & 131.4 & $21 \cdot 0$ & $134 \cdot 3$ & 19.5 & & \\
\hline \multicolumn{9}{|l|}{ Observe } \\
\hline Face & $26 \cdot 2$ & $5 \cdot 1$ & $27 \cdot 3$ & $3 \cdot 8$ & 28.0 & 5.0 & 0.24 & $0.002^{\star \star}, \dagger$ \\
\hline Mobile & $26 \cdot 0$ & 5.0 & $27 \cdot 2$ & 5.4 & 27.4 & 6.4 & $0 \cdot 16$ & \\
\hline Control & $26 \cdot 0$ & $5 \cdot 0$ & $25 \cdot 6$ & $5 \cdot 6$ & $26 \cdot 6$ & $5 \cdot 3$ & & \\
\hline \multicolumn{9}{|l|}{ Describe } \\
\hline Face & 29.4 & $6 \cdot 5$ & $30 \cdot 0$ & $7 \cdot 0$ & $30 \cdot 6$ & $5 \cdot 6$ & 0.15 & 0.128 \\
\hline Mobile & 29.8 & $6 \cdot 8$ & 31.0 & $6 \cdot 8$ & 30.4 & $7 \cdot 0$ & 0.06 & \\
\hline Control & $30 \cdot 2$ & $6 \cdot 7$ & $29 \cdot 8$ & $6 \cdot 6$ & $30 \cdot 4$ & $6 \cdot 3$ & & \\
\hline \multicolumn{9}{|l|}{ ActAware } \\
\hline Face & $27 \cdot 7$ & 5.9 & $26 \cdot 2$ & $5 \cdot 8$ & $27 \cdot 3$ & $5 \cdot 3$ & -0.10 & $0.107 \ddagger / 0.251 \S$ \\
\hline Mobile & $25 \cdot 3$ & $5 \cdot 6$ & $25 \cdot 8$ & $6 \cdot 3$ & 27.0 & 6.8 & 0.26 & \\
\hline Control & $25 \cdot 1$ & $5 \cdot 9$ & $25 \cdot 2$ & 6.5 & $25 \cdot 3$ & $6 \cdot 1$ & & \\
\hline \multicolumn{9}{|l|}{ NonJudge } \\
\hline Face & 28.9 & $6 \cdot 1$ & $28 \cdot 3$ & $6 \cdot 1$ & $30 \cdot 0$ & $5 \cdot 3$ & -0.03 & 0.389 \\
\hline Mobile & 28.6 & $6 \cdot 2$ & 29.9 & $5 \cdot 3$ & 31.6 & 5.4 & 0.26 & \\
\hline Control & $28 \cdot 1$ & $7 \cdot 2$ & 28.6 & $7 \cdot 0$ & 29.4 & $7 \cdot 2$ & & \\
\hline \multicolumn{9}{|l|}{ NonReact } \\
\hline Face & $22 \cdot 2$ & $4 \cdot 3$ & $22 \cdot 2$ & 4.6 & 23.0 & 4.8 & -0.02 & $0.631 \ddagger / 0.161 \S$ \\
\hline Mobile & 21.9 & $5 \cdot 2$ & 23.4 & 4.6 & 23.3 & $5 \cdot 3$ & 0.10 & \\
\hline Control & $21 \cdot 7$ & 5.5 & $22 \cdot 1$ & $5 \cdot 4$ & $22 \cdot 6$ & $5 \cdot 0$ & & \\
\hline \multicolumn{9}{|l|}{ SOC-13 } \\
\hline Face & $53 \cdot 1$ & $5 \cdot 1$ & $62 \cdot 6$ & 11.7 & 63.8 & 11.0 & 0.14 & 0.525 \\
\hline Mobile & 51.9 & $5 \cdot 3$ & 61.6 & 11.0 & 63.6 & 11.6 & 0.32 & \\
\hline Control & $51 \cdot 1$ & $6 \cdot 3$ & 59.6 & $12 \cdot 0$ & 61.0 & $12 \cdot 7$ & & \\
\hline
\end{tabular}

Pre, pre-intervention; post, post-intervention; fup, follow-up; AAQ-II, Acceptance and Action Questionnaire II; AAQW, Acceptance and Action Questionnaire for Weight; FFMQ, Five Facet Mindfulness Questionnaire; Observe, Observing subscale of FFMQ; Describe, Describing subscale of FFMQ; ActAware, Acting with Awareness subscale of FFMQ; NonJudge, Non-judgement of Inner Experiences subscale of FFQM; NonReact, Non-reactivity to Inner Experiences subscale of FFMQ; SOC-13, sense of coherence measured with the thirteen-item Orientation to Life Questionnaire.

Means and SD were calculated using Mplus software version 7 with full information maximum likelihood estimates for the parameters. A between-group effect size of 0.2 was considered clinically small, 0.5 medium and 0.8 large ${ }^{(45)}$.

${ }^{\star *} P<0.01$.

†Difference in change scores from pre to post was significance at the $P<0.05$ level. Difference in change scores from post to fup was not significant at the $P<0.05$ level.

‡Between-group analysis comparing ACT face-to-face with the control group.

$\S$ Between-group analysis comparing ACT mobile with the control group.

presented in Table 4. There were no statistically significant direct effects $(c ; P<0 \cdot 05$; see Fig. 1$)$ when the indirect path ( $a$ and $b$ ) was included in the model, indicating indirectonly mediation concerning all significant indirect effects ${ }^{(48)}$. The estimates for statistically significant indirect effects ranged from $0 \cdot 05$ to $0 \cdot 08$, indicating small effects (effect size $>0 \cdot 02^{(45)}$ ).

Mindfulness skills (FFMQ and its subscales), general psychological flexibility (AAQ-II) and sense of coherence (SOC-13) did not mediate any intervention effects on intuitive eating and weight.

\section{Discussion}

The present study is one of the first to examine processes of change in psychological approaches to adaptive eating behaviour and weight management. Despite the promising results related to intuitive eating, few studies have attempted to explain the processes encouraging adaptive eating behaviour and previous studies on intuitive eating have been mainly observational in nature. Thus, the focus of the present study was on exploring mechanisms of change in intuitive eating and weight in the ACT 
Table 4 Unstandardized regression coefficients $a, b$ and $c$ (and $P$ values) of the mediation models in which the Acceptance and Action Questionnaire for Weight (AAQW; pre-post) mediated the effect of the acceptance and commitment therapy Interventions on the outcome variables

\begin{tabular}{lcrr}
\hline $\begin{array}{l}\text { Outcome variable } \\
\text { (pre-fup) }\end{array}$ & a/P value & \multicolumn{1}{c}{$b / P$ value } & \multicolumn{1}{c}{$c / P$ value } \\
\hline BMI & $-2 \cdot 369 / 0.000$ & $0.091 / 0.000$ & $-0.086 / 0.591$ \\
IES total & $-2 \cdot 391 / 0.000$ & $-0.125 / 0.015$ & $0.201 / 0.510$ \\
IES: Reasons & $-2 \cdot 350 / 0.000$ & $-0.042 / 0.021$ & $0.122 / 0.264$ \\
IES: Cues & $-2 \cdot 393 / 0.000$ & $-0.065 / 0.003$ & $-0.111 / 0.461$
\end{tabular}

Pre, pre-intervention; post, post-intervention; fup, follow-up; IES, Intuitive Eating Scale; Reasons, Eating for Physical rather than Emotional Reasons subscale; Cues, Reliance on Internal Hunger and Satiety Cues subscale.

intervention groups compared with the control group of participants having overweight.

First, the present results indicate that Eating for Physical rather than Emotional Reasons increased in the ACT-based intervention groups. The present findings support the theoretical assumption that ACT reduces avoidance behaviour, such as emotional eating. Considering the association between emotional eating and obesity ${ }^{(13)}$, these results suggest that ACT-oriented interventions can have a positive impact on weight management and disordered eating. These results are in line with previous studies that have indicated that mindfulness practice reduces emotional and external eating ${ }^{(31)}$, as well as binge eating ${ }^{(52)}$. In the present study, the ACT mobile intervention also improved mindfulness skills (FFMQ total) and both ACT interventions enhanced Observing (subscale of FFMQ).

Second, the mediation analysis indicated that the intervention effects on weight and intuitive eating behaviours, Eating for Physical rather than Emotional Reasons and Reliance on Internal Hunger and Satiety Cues were mediated by change in weight-related psychological flexibility (AAQW) in the ACT interventions. Thus, the ACT interventions increased psychological flexibility (AAQW), which in turn promoted weight loss and eating based on bodily cues of hunger and satiety. In contrast, weightrelated psychological flexibility was not found to mediate the interventions' effect on Unconditional Permission to Eat. This may be due to the fact that ACT interventions emphasized more mindful eating than content reflected by the subscale of Unconditional Permission to Eat. Moreover, it could be speculated that ACT-based interventions might change the impact of emotional reactions to eating patterns.

The present findings support the idea that ACT-oriented interventions for lifestyle changes improve individuals' ability to continue with valued activities even when confronted with negative emotions and thoughts related to weight, and this process mediated changes in eating regulation and weight. In accordance with our findings, population-specific measures of psychological flexibility have been significant mediators in many different areas, 
including with respect to smoking cessation ${ }^{(23)}$, diabetes self-care $^{(24)}$, prejudice ${ }^{(27)}$, seizures and quality of life in epilepsy cases ${ }^{(25)}$, adaptive functioning in chronic pain patients ${ }^{(53-55)}$ and weight-related issues ${ }^{(18,56)}$.

Mindfulness skills (FFMQ) and sense of coherence (SOC-13), as well as the general measure of psychological flexibility (AAQ-II), were not found to mediate changes in weight or intuitive eating behaviour. These measures (FFMQ, SOC-13, AAQ-II) are not specific to weight or to eating issues. Thus, this might imply that change processes associated with weight management and eating regulation are better assessed by specific measures targeted to describe weight-related thoughts, feelings and actions. Further, the fact that weight-specific psychological flexibility mediated weight change and intuitive eating might suggest that interventions should target weight-specific behavioural patterns (such as the avoidance of feelings related to weight and body image) and they should include specific exercises or skill training aimed at dealing with weight-related avoidance.

Correspondingly, Webb and Hardin found that body image flexibility, reflecting the willingness to approach aversive body image-related internal content with mindful acceptance in order to continue to move in valued life directions, partially mediated the relationship between adhering to internalized weight-stigmatizing beliefs and the likelihood of practising intuitive eating in a weightdiverse sample of college women ${ }^{(57)}$. Body image flexibility has also explained the relationship between selfcompassion and intuitive eating ${ }^{(58)}$. Together with our results, these findings suggest that the willingness to approach aversive thoughts, emotions and physiological sensations regarding the body with mindful acceptance may have an important role in promoting intuitive eating (i.e. values-driven behaviour).

The promise of mediation analysis is that it can identify fundamental processes underlying human behaviour that are relevant across behaviours and contexts. The present study found similar intervention effects and mediating processes for the ACT mobile stand-alone intervention (minimal contact) as for the ACT face-to-face group intervention, which supports the assumption that these particular behavioural processes are essential in supporting adaptive eating attitudes and weight management. In regard to affecting psychological flexibility and mindfulness skills, these results also suggest that skill training plays a more important role than face-to-face coaching. This hypothesis needs to be tested in future studies.

\section{Limitations}

The most significant limitation of the current study is that the processes were measured partly in parallel. Thus, the temporal precedence of the mediator cannot be established, making it difficult to separate cause and effect. Unfortunately, a lack of assessment points during the active treatment phase precluded a detailed examination of the directionality of change. Moreover, the time periods of the present study, about 2 months for the process measurements and 8 months for the outcomes, may have been too short for some mediation effects to take place. For example, adopting mindfulness skills and utilizing them to improve eating behaviours and increase weight loss more significantly may need more time.

Another significant limitation of our study is the use of self-report measures to assess the psychological constructs of interest. Thus, we cannot be sure that observed changes in processes actually reflect 'true' changes in target constructs - they may simply reflect social demand or expectations of the treatment. It is also important to note that intuitive eating is a cognitive construct that may be related to eating habits, but more studies are needed to verify that. The present results support this connection by showing similar mediation effects on both BMI and intuitive eating. Still, future studies should aim to include behavioural, experimental and observer-rated processes of acceptance/experiential avoidance and eating behaviour. Also, a variety of approaches have been proposed for the assessment of mindfulness and there have been debates about the issue of measuring this construct ${ }^{(59,60)}$. Thus, further research using other validated measures of mindfulness is recommended.

Moreover, the inclusion of multiple mediation analyses leads to an increased risk of type 1 error. However, AAQW mediated intervention effects systemically on all outcome variables, except for Unconditional Permission to Eat, whereas other process variables (AAQ-II, SOC-13, FFMQ and its subscales) did not have any significant indirect effects. Moreover, it is important to note that the observed effect sizes of the intervention effects as well as the indirect effects were small, and thus the clinical significance of the observations is unclear. Further research is warranted to investigate the effects and mechanisms of ACT-based interventions on eating behaviours. There are possibly also other important variables mediating intervention effects on intuitive eating and weight. Finally, it is important to note that our sample consisted predominantly of overweight women experiencing psychological stress, who were willing to make changes to improve their lifestyle. The participants were also relatively highly educated. Therefore, the generalizability of the results to the other populations must be tested in future studies.

\section{Conclusions}

In conclusion, our research provides evidence that ACToriented interventions might improve overweight persons' skills in dealing with emotions related to eating. Our data also imply that weight-related psychological flexibility mediates changes in weight and intuitive eating, especially in eating based on physical reasons and reliance on hunger and satiety cues, in overweight persons experiencing psychological stress. Thus, these findings support 
the idea that ACT-oriented interventions for lifestyle changes improve individuals' ability to continue with valued activities even when confronted with negative emotions and thoughts related to weight, and this process mediates changes in eating regulation and weight. More generally, the present results suggest that one way to increase adaptive eating behaviour is to enhance psychological flexibility related to weight issues. Studies are needed to investigate whether interventions targeted to enhance weight-related psychological flexibility lead to better long-term weight maintenance as compared with current weight-loss programmes.

\section{Acknowledgements}

Financial support: This study was supported by the SalWe Research Program for Mind and Body (Tekes - the Finnish Funding Agency for Technology and Innovation; grant number 1104/10). Tekes had no role in the design, analysis or writing of this article. Conflict of interest: The authors have no competing interests to declare. Authorship: All authors participated in designing the study, carrying it out and commented on the manuscript. A.T. and E.S. participated in doing statistical analysis. E.S. and R.L. had the main responsibility in writing the article and formulating the research questions. Ethics of buman subject participation: This study was conducted according to the guidelines laid down in the Declaration of Helsinki and all procedures involving human subjects were approved by the Ethics Committee of the Central Finland Health Care District. Written informed consent was obtained from all subjects/patients.

\section{References}

1. Jeffery RW, Epstein LH, Wilson GT et al. (2000) Long-term maintenance of weight loss: current status. Health Psychol 19, Suppl. 1, 5-16.

2. Mann T, Tomiyama AJ, Westling E et al. (2007) Medicare's search for effective obesity treatments: diets are not the answer. Am Psychol 62, 220-233.

3. Garner DM \& Wooley SC (1991) Confronting the failure of behavioral and dietary treatments for obesity. Clin Psychol Rev 11, 729-780.

4. Wing RR \& Phelan S (2005) Long-term weight loss maintenance. Am J Clin Nutr 82, 222-225.

5. Avalos L \& Tylka T (2006) Exploring a model of intuitive eating with college women. J Couns Psychol 53, 486-497.

6. Tylka T (2006) Development and psychometric evaluation of a measure of intuitive eating. J Couns Psychol 53, 226-240.

7. Bacon L, Stern J, Van Loan M et al. (2005) Size acceptance and intuitive eating improve health for obese, female chronic dieters. J Am Diet Assoc 105, 929-936.

8. Augustus-Horvath C \& Tylka T (2011) The acceptance model of intuitive eating: a comparison of women in emerging adulthood, early adulthood, and middle adulthood. J Couns Psychol 58, 110-125.

9. Hawks S, Madanat H, Hawks J et al. (2005) The relationship between intuitive eating and health indicators among college women. Am J Health Educ 36, 331-336.

10. Forman EM \& Butryn ML (2015) A new look at the science of weight control: how acceptance and commitment strategies can address the challenge of self-regulation. Appetite 84, 171-180.

11. Castelnuovo-Tedesco P \& Schiebel D (1975) Studies of superobesity: I. Psychological characteristics of superobese patients. Int J Psychiatry Med 6, 465-480.

12. Hockley RE (1979) Toward an understanding of the obese person. J Relig Health 18, 120-131.

13. Hudson A \& Williams SG (1981) Eating behavior, emotions, and overweight. Psychol Rep 48, 669-670.

14. Hayes SC, Luoma JB, Bond FW et al. (2006) Acceptance and commitment therapy: model, processes and outcomes. Behav Res Ther 44, 1-25.

15. Lillis J \& Hayes SC (2008) Measuring avoidance and inflexibility in weight related problems. Int J Behav Consult Ther 4, 348-354.

16. Forman EM, Hoffman KL, McGrath KB et al. (2007) A comparison of acceptance- and control-based strategies for coping with food cravings: an analog study. Behav Res Ther 45, 2372-2386.

17. Hooper N, Sandoz EK, Ashton J et al. (2012) Comparing thought suppression and acceptance as coping techniques for food cravings. Eating Behav 13, 62-64.

18. Lillis J, Hayes SC, Bunting K et al. (2009) Teaching acceptance and mindfulness to improve the lives of obese: a preliminary test of a theoretical model. Ann Behav Med 37, 58-69.

19. Hayes SC, Strosahl K \& Wilson KG (editors) (1999) Acceptance and Commitment Therapy: An Experiential Approach to Behavior Change. New York: Guilford Press.

20. Kashdan T \& Rottenberg J (2010) Psychological flexibility as a fundamental aspect of health. Clin Psychol Rev 30, 865-878.

21. Brown KW \& Ryan RM (2003) The benefits of being present: mindfulness and its role in psychological well-being. J Pers Soc Psychol 84, 822-848.

22. Chambers R, Gullone E \& Allen NB (2009) Mindful emotion regulation: an integrative review. Clin Psychol Rev 29, 560-572.

23. Gifford EV, Kohlenberg BS, Hayes SC et al. (2004) Acceptance-based treatment for smoking cessation. Behav Ther 35, 689-705.

24. Gregg JA, Callaghan GM, Hayes SC et al. (2007) Improving diabetes self-management through acceptance, mindfulness, and values: a randomized controlled trial. J Consult Clin Psychol 75, 336-343.

25. Lundgren T, Dahl J \& Hayes SC (2008) Evaluation of mediators of change in the treatment of epilepsy with acceptance and commitment therapy. J Behav Med 31, 225-235.

26. Lappalainen R, Lehtonen T, Skarp E et al. (2007) The impact of CBT and ACT models using psychology trainee therapists: a preliminary controlled effectiveness trial. Behav Modif 31, 488-511.

27. Lillis J \& Hayes SC (2007) Applying acceptance, mindfulness, and values to the reduction of prejudice: a pilot study. Behav Modif 31, 389-411.

28. Varra AA, Hayes SC, Roget N et al. (2008) A randomized control trial examining the effect of acceptance and commitment training on clinician willingness to use evidence-based pharmacotherapy. J Consult Clin Psychol 76, 449-458.

29. Zettle RD \& Hayes SC (1986) Dysfunctional control by client verbal behavior: the context of reason-giving. Anal Verbal Behav 4, 30-38.

30. Kristeller J \& Wolever R (2011) Mindfulness-based eating awareness treatment (MB-EAT): conceptual basis. Eat Disord 19, 49-61.

31. Alberts H, Thewissen R \& Raes L (2012) Dealing with problematic eating bahaviour: the effects of a mindfulnessbased intervention on eating behavior, food cravings, dichotomous thinking and body image concern. Appetite 58, $847-851$. 
32. Albers S (2011) Using mindful eating to treat food restriction. a case study. Eat Disord 19, 97-107.

33. Lappalainen R, Sairanen E, Järvelä E et al. (2014) The effectiveness and applicability of different lifestyle interventions for enhancing wellbeing: the study design for a randomized controlled trial for persons with metabolic syndrome risk factors and psychological distress. BMC Public Health 14, 310.

34. Eriksson M \& Lindstrom B (2005) Validity of Antonovsky's sense of coherence scale: a systematic review. J Epidemiol Community Health 59, 460-466.

35. Makowska Z, Merecz D, Moscicka A et al. (2002) The validity of general health questionnaires, GHQ-12 and GHQ-28, in mental health studies of working people. Int J Occup Med Environ Health 15, 353-362.

36. Ahtinen A, Mattila E, Välkkynen P et al. (2013) Mobile mental wellness training for stress management: feasibility and design implications based on a one-month field study. JMIR mHealth uHealth 1, e11.

37. Lattimore P, Fisher N \& Malinowski P (2011) A crosssectional investigation of trait disinhibition and its association with mindfulness and impulsivity. Appetite 56, 241-248.

38. Bond FW, Hayes SC, Baer RA et al. (2011) Preliminary psychometric properties of the Acceptance and Action Questionnaire-II: a revised measure of psychological inflexibility and experiential avoidance. Behav Ther 42, 676-688.

39. Antonovsky A (1993) The structure and properties of the sense of coherence scale. Soc Sci Med 36, 725-733.

40. Antonovsky A (1987) Unraveling the Mystery of Health: How People Manage Stress and Stay Well. San Francisco, CA: Jossey-Bass.

41. Suominen S, Blomberc H, Helenius H et al. (1999) Sense of coherence and health - does the association depend on resistance resources? A study of 3115 adults in Finland. Psychol Health 14, 937-948.

42. Suominen S, Helenius H, Blomberg H et al. (2001) Sense of coherence as a predictor of subjective state of health: results of 4 years of follow-up of adults. J Psychosom Res 50, 77-86.

43. Sairanen E, Tolvanen A, Karhunen L et al. (2015) Psychological flexibility and mindfulness explain intuitive eating in overweight adults. Behav Modif 39, 557-579.

44. Lappalainen P, Langrial S, Oinas-Kukkonen H et al. (2015) Web-based acceptance and commitment therapy for depressive symptoms with minimal support a randomized controlled trial. Behav Modif 39, 805-834.

45. Cohen J (1992) A power primer. Psychol Bull 112, 155-159.

46. MacKinnon DP (2008) Introduction to Statistical Mediation Analysis. New York: Routledge.
47. Russell DW, Kahn JH, Spoth R et al. (1998) Analyzing data from experimental studies: a latent variable structural equation modeling approach. J Couns Psychol 45, 18-29.

48. Zhao X, Lynch JG \& Chen Q (2010) Reconsidering Baron and Kenny: myths and truths about mediation analysis. J Consum Res 37, 197-206.

49. MacKinnon DP, Lockwood CM, Hoffman JM et al. (2002) A comparison of methods to test mediation and other intervening variable effects. Psychol Methods 7, 83.

50. Muthén BO (1998-2004) Appendix 5. In Mplus Technical Appendices. Los Angeles, CA: Muthén \& Muthén.

51. Hu L \& Bentler PM (1999) Cutoff criteria for fit indexes in covariance structure analysis: conventional criteria versus new alternatives. Struct Equ Modeling 6, 1-55.

52. Kristeller J \& Hallett B (1999) Effects of a meditation-based intervention in the treatment of binge eating. $J$ Health Psychol 4, 357-363.

53. McCracken LM, Vowles KE \& Eccleston C (2005) Acceptance-based treatment for persons with complex, long standing chronic pain: a preliminary analysis of treatment outcome in comparison to a waiting phase. Behav Res Ther 43, 1335-1346.

54. Vowles KE \& McCracken LM (2008) Acceptance and values-based action in chronic pain: a study of treatment effectiveness and process. J Consult Clin Psychol 76, 397-407.

55. Wicksell RK, Ahlqvist J, Bring A et al. (2008) Can exposure and acceptance strategies improve functioning and life satisfaction in people with chronic pain and whiplashassociated disorders (WAD)? A randomized controlled trial. Cogn Behav Ther 37, 169-182.

56. Weineland S, Hayes SC \& Dahl J (2012) Psychological flexibility and the gains of acceptance-based treatment for post-bariatric surgery: six-month follow-up and a test of the underlying model. Clin Obes 2, 15-24.

57. Webb JB \& Hardin AS (2016) An integrative affect regulation process model of internalized weight bias and intuitive eating in college women. Appetite 102, 60-69.

58. Schoenefeld SJ \& Webb JB (2013) Self-compassion and intuitive eating in college women: examining the contributions of distress tolerance and body image acceptance and action. Eat Behav 14, 493-496.

59. Bishop SR, Lau M, Shapiro S et al. (2004) Mindfulness: a proposed operational definition. Clin Psychol 11, 230-241.

60. Brown KW \& Ryan RM (2004) Perils and promise in defining and measuring mindfulness: observations from experience. Clin Psychol 11, 242-248. 